\title{
ASO Author Reflections: Making a Wide Window to the Hepatocaval Confluence for Laparoscopic Resection of a Deep Segment 8 Lesion
}

\author{
Satoshi Ogiso, MD, PhD, FACS, and Satoru Seo, MD, PhD \\ Division of Hepato-Biliary-Pancreatic Surgery and Transplantation, Department of Surgery, Graduate School of Medicine, \\ Kyoto University, Kyoto, Japan
}

\section{PAST}

Resection of liver lesions contacting the hepatocaval confluence poses a risk of injury to major hepatic veins/ vena cava, which potentially leads to massive bleeding as well as fatal gas embolism when laparoscopy is performed. Although meticulous dissection is needed to detach lesions from major hepatic veins/vena cava, the laparoscopic view and access to the hepatocaval confluence is significantly limited. ${ }^{1,2}$ Therefore, this difficult resection remains a nonoptimal candidate for laparoscopy.

\section{PRESENT}

Even in open hepatectomy, limited resection of deep or large segment 8 lesions disrupts the surgical field at the hepatocaval confluence. Then a conventional transfissural approach is effectively used to open the midplane of the liver widely along the middle hepatic vein $(\mathrm{MHV})^{3,4}$ and obtain an improved surgical field at the hepatocaval confluence. This approach was applied to laparoscopic segment 8 resection for lesions contacting the hepatocaval confluence, suggesting that the approach is useful for facilitating such a demanding procedure. ${ }^{5}$

\section{FUTURE}

Laparoscopic segment 8 resection can be safely disseminated by using a transfissural approach, even when lesions are large or deeply located and in contact with the hepatocaval confluence. Further studies are warranted to accumulate the necessary data and confirm the reproducibility and safety of the technique.

DISCLOSURE There are no conflict of interest.

\section{REFERENCES}

1. Ogiso S, Conrad C, Araki K, et al. Laparoscopic transabdominal with transdiaphragmatic access improves resection of difficult posterosuperior liver lesions. Ann Surg. 2015;262:358-65.

2. Ogiso S, Nomi T, Araki K, et al. Laparoscopy-specific surgical concepts for hepatectomy based on the laparoscopic caudal view: a key to reboot surgeons' minds. Ann Surg Oncol. 2015;22(Suppl 3):S327-33.

3. Couinaud C. Surgical anatomy of the liver revisited. C. Couinaud, Paris; 1989.

4. Honda G, Kurata M, Tsuruta K. Approach for systematic resection of the liver anterosuperior area: exposing Glissonean pedicles by prior dissection of the major hepatic fissure. J Am Coll Surg. 2008;207:e1-4.

5. Ogiso S, Seo S, Ishii $\mathrm{T}$, et al. Transfissural approach for laparoscopic resection of a deep segment 8 lesion in contact with the hepatocaval confluence. Ann Surg Oncol. 2020. https://doi.org/ 10.1245/s10434-020-09309-5.

Publisher's Note Springer Nature remains neutral with regard to jurisdictional claims in published maps and institutional affiliations.

(C) Society of Surgical Oncology 2021

First Received: 26 October 2020

Accepted: 27 October 2020;

Published Online: 3 January 2021

S. Seo, MD, PhD

e-mail: rutosa@kuhp.kyoto-u.ac.jp 